Pacific Journal of Mathematics

STRONG QUASI-CONVEXITY 


\title{
STRONG QUASI-CONVEXITY
}

\author{
EDWARD SilvermaN
}

\begin{abstract}
Quasi-convexity and strong quasi-convexity are conditions on integrands of multiple integrals which are associated with lower semi-continuity. Equivalent definitions are given which, at least for parametric integrands, are more geometric in character than the original definitions.
\end{abstract}

About twenty years ago Morrey derived a necessary condition, strong quasi-convexity, for the lower semi-continuity of certain multiple integrals [2, 3, p. 113]. Morrey also obtained conditions under which strong quasi-convexity is equivalent to quasi-convexity, a differentialless analogue of the Legendre-Hadamard condition. We will use an idea of Cesari to rewrite Morrey's condition to make it more closely resemble the usual definition of convexity, and will then extend Morrey's results relating 'convexity in the Jacobians' with quasi-convexity [3, p. 124].

Let $N \geqq \nu$ be natural numbers, let $E=R^{N}$ and let

$$
P=L\left(R^{\nu}, E\right) \approx E^{\nu} .
$$

Let $f$ be real-valued and continuous on $R^{\nu} \times E \times P$ and let $G$ and $H$ always denote bounded open subsets of $R^{\nu}$.

If $z \in C(G, E)$ and if $z$ is Lipschitzian let

$$
I_{G}(z)=\int_{G} f\left(x, z(x), z^{\prime}(x)\right) d x .
$$

If $I_{G}(z) \leqq I_{G}(w)$ whenever $w$ is Lipschitzian and $\|w-z\|$ is sufficiently small, then $z$ furnishes $I_{G}$ with a relative minimum. If $I_{G}$ is lower semicontinuous for each $G$, then $I$ is said to be lower semicontinuous.

If $A$ is an open set, or the closure of an open set in $R^{\nu}$, let $|A|$ be its $\nu$-dim Lebesgue measure.

Part (ii) of the following theorem is weaker than [3, p. 113] and the proof of (i) is, except for a trivial modification, the same as that for part (ii).

THEOREM 1. Let $\zeta$ be Lipschitzian on $G$ into $E$ and vanish on $\partial G$.

(i) If $z \in C^{\prime}(H, E)$ furnishes $I_{H}$ with a relative minimum and if $y \in G \cap H$ then 


$$
\int_{G} f\left(y, z(y), z^{\prime}(y)+\zeta^{\prime}(u)\right) d u \geqq f\left(y, z(y), z^{\prime}(y)\right)|G| \cdot
$$
then

(ii) If $I$ is lower semicontinuous, if $y \in R^{\nu}, w \in E$ and $p \in P$,

$$
\int_{G} f\left(y, w, p+\zeta^{\prime}(u)\right) d u \geqq f(y, w, p)|G| \cdot
$$

We notice that the proof makes use of the fact that almost all points of $\partial G$ are points of density for Comp $G$ so that $\zeta^{\prime}(x)=0$ for almost all $x$ in $\partial G$. This fact is also used in the proof of Theorem 2.

By definition, [3, p. 114], $f$ is strongly quasi-convex if for each bounded open set $G$ in $R^{\nu}$, for each $y \in G, w \in E$ and $p \in P$,

$$
\int_{G} f\left(y, w, p+\zeta^{\prime}(x)\right) d x \geqq f(y, w, p)|G|
$$

whenever $\zeta$ is Lipschitzian on $G$ into $E$ and vanishes on $\partial G$.

Let 3 be the collection of all quasilinear functions on $R^{\nu}$ into $E$ which have compact support. If $\zeta \in 3$ let $\Delta(\zeta)$ be the collection of simplexes on each of which $\zeta$ is linear and whose union is $K_{\zeta}=$ support $\zeta$. If $\delta \in \Delta(\zeta)$ let $\zeta^{\prime}(\delta)=\zeta^{\prime}(u)$ for any $u \in \operatorname{Int} \delta$.

THEOREM 2. A necessary and sufficient condition that $f$ be strongly quasi-convex is that

$$
f(y, w, p) \leqq \sum_{\delta \in \Delta(\zeta)} \frac{|\delta|}{\left|K_{\zeta}\right|} f\left(y, w, p+\zeta^{\prime}(\delta)\right)
$$

for each $\zeta \in 3, y \in R$, $w \in E$ and $p \in P$.

The necessity is an immediate consequence of the definitions, while the sufficiency results from the fact that 3 is big enough to approximate all Lipschitzian functions with compact support.

LEMMA 1. Let $\phi$ be quasilinear on a polyhedral region $\pi \subset R^{\nu}$ into $R^{\nu}$. If $\phi^{i}$ vanishes on $\partial \pi$ for some $i=1, \cdots, \nu$ then

$$
\int_{\pi} \frac{d \phi}{d u} d u=0
$$

The integral is equal to the integral of the topological index, which is identically zero since $\phi(\partial \pi)$ is contained in a hyperplane.

Let $\Lambda: E^{\nu} \rightarrow \wedge^{\nu} E$ be defined by $\Lambda(p)=p_{1} \wedge \cdots \wedge p_{\nu}$ if

$$
p_{1}, \cdots, p_{\nu} \in E \text {. }
$$


Lemma 2. Let $p \in P$ and suppose that $\zeta \in 3$. Then

$$
\frac{1}{\left|K_{\zeta}\right|} \sum_{\delta \in \Delta(\zeta)} \Lambda\left(p+\zeta^{\prime}(\delta)\right)|\delta|=\Lambda(p) \text {. }
$$

Proof. Let $1 \leqq \lambda_{1}<\cdots<\lambda_{\nu} \leqq N$. If $\alpha \in \wedge^{\nu} E$ let $\alpha^{\lambda}=\alpha^{\lambda_{1} \cdots \lambda_{\nu}}$. If $\phi$ maps a set $S$ into $E$ let $\phi^{\lambda}=\left(\phi^{\lambda_{1}}, \cdots, \phi^{\lambda_{\nu}}\right)$. If

$$
x=p_{1} u^{1}+\cdots+p_{\nu} u^{\nu}
$$

then $d x / d u=p$. Thus

$$
\begin{aligned}
\sum_{\delta \in \Delta(\zeta)} \Lambda\left(p+\zeta^{\prime}(\delta)\right)^{\lambda}|\delta| & =\int_{K_{\zeta}} \frac{d\left(x^{\lambda}+\zeta^{\lambda}\right)}{d u} d u \\
& =\int_{K_{\zeta}} \frac{d x^{\lambda}}{d u} d u=\Lambda(p)^{\lambda}\left|K_{\zeta}\right|
\end{aligned}
$$

by Lemma 1 and a standard expansion of the determinant

$$
\frac{d\left(x^{\lambda}+\zeta^{\lambda}\right)}{d u}
$$

The lemma follows.

Let $F$ be real-valued and continuous on $\wedge^{\nu} E$. Then $F$ is said to be almost convex ${ }^{1}$ if

$$
F(\Lambda(p)) \leqq \sum_{\delta \in \Delta(\zeta)} \frac{|\delta|}{\left|K_{\zeta}\right|} F\left(\Lambda\left(p+\zeta^{\prime}(\delta)\right)\right.
$$

for each $p \in E^{\nu}$ and $\zeta \in 3$. If $F$ is convex then, by Lemma 2,

$$
\begin{aligned}
F(\Lambda(p)) & =F\left(\frac{1}{\left|K_{\zeta}\right|} \sum_{\delta \in \Delta(\zeta)} \Lambda\left(p+\zeta^{\prime}(\delta)\right)|\delta|\right) \\
& \leqq \sum_{\delta \in \Delta(\zeta)} \frac{|\delta|}{\left|K_{\zeta}\right|} F\left(\Lambda\left(p+\zeta^{\prime}(\delta)\right)\right)
\end{aligned}
$$

so that $F$ is almost convex.

THEOREM 3. Let $F$ be real-valued and continuous on $\wedge^{\nu} E$. Then $F \circ \Lambda$ is strongly quasi-convex if and only if $F$ is almost convex.

Proof. Let $F$ be almost convex. If $f=F \circ \Lambda$ and $\zeta \in 3$, then by Lemma 2

\footnotetext{
1 This definition differs slightly from that in $[\mathbf{1}$, p. 30].
} 


$$
\begin{aligned}
f(p) & =F\left(\frac{1}{\left|K_{\zeta}\right|} \sum_{\delta \in \Delta(\zeta)} \Lambda\left(p+\zeta^{\prime}(\delta)\right)|\delta|\right) \\
& \leqq \sum_{\delta \in \Delta(\zeta)} \frac{|\delta|}{\left|K_{\zeta}\right|} F\left(\Lambda\left(p+\zeta^{\prime}(\delta)\right)\right) \\
& =\sum_{\delta \in \Delta(\zeta)} \frac{|\delta|}{\left|K_{\zeta}\right|} f\left(p+\zeta^{\prime}(\delta)\right)
\end{aligned}
$$

so that $f$ is strongly quasi-convex.

Now suppose $f$ is strongly quasi-convex. A repetition of the above argument gives

$$
\begin{aligned}
F(\Lambda(p)) & =f(p) \\
& \leqq \sum_{\delta \in \Delta(\zeta)} \frac{|\delta|}{\left|K_{\zeta}\right|} f\left(p+\zeta^{\prime}(\delta)\right) \\
& =\sum_{\delta \in \Delta(\zeta)} \frac{|\delta|}{\left|K_{\zeta}\right|} F\left(\Lambda\left(p+\zeta^{\prime}(\delta)\right)\right)
\end{aligned}
$$

so that $F$ is almost convex.

To study the notion of quasi-convexity it is convenient to use the following notation:

$$
\text { if } p \in P, \lambda \in R_{\nu}=\left(R^{\nu}\right)^{\prime} \text { and } \xi \in E,
$$

let $p+\lambda \xi \in P$ be defined by

$$
(p+\lambda \xi)_{\alpha}^{i}=p_{\alpha}^{i}+\lambda_{\alpha} \xi^{i}
$$

$i=1, \cdots, N$ and $\alpha=1, \cdots, \nu$.

We say that $f$ is quasi-convex [2,p.112] if

(i) for fixed $p$ and $\xi$, $\phi$ is convex over $R_{\nu}$ where

$$
\phi(\lambda)=f(p+\lambda \xi) \text {, }
$$

and

(ii) for fixed $p$ and $\lambda$, $\psi$ is convex over $E$ where

$$
\psi(\xi)=f(p+\lambda \xi) \text {. }
$$

If $f$ is strongly quasi-convex, then $f$ is quasi-convex [2, p. 114].

THEOREM 4. The following statements are equivalent:

(a) $f$ is quasi-convex, and

(b) if $p$ and $\xi$ are fixed and $\phi(\lambda)=f(p+\lambda \xi)$ then $\phi$ is convex,

(c) if $p$ and $\lambda$ are fixed and $\psi(\xi)=f(p+\lambda \xi)$ then $\psi$ is convex.

Proof. Suppose that (b) holds. Let $p$ and $\lambda$ be fixed. Now let $\xi$ and $\eta$ be in $E$ and $t \in(0,1)$. Let $q=p+\lambda(t \xi+(1-t) \eta)$, let 
$\zeta=\eta-\xi$ and let $\phi(\mu)=f(q+\mu \zeta)$. Since $\phi$ is convex and

$$
\begin{gathered}
(1-t)(t \lambda)+t(-(1-t) \lambda)=0, \\
f(q)=\phi(0) \\
=t \phi(-(1-t) \lambda)+(1-t) \dot{\phi}(t \lambda) \\
=t f(p+\lambda \xi)+(1-t) f(p+\lambda \eta) .
\end{gathered}
$$

Hence (a) and (b) are equivalent. A dual argument shows that (a) and (c) are also equivalent.

Since quasi-convexity of $f$ depends only upon the behavior of $f$ relative to its third argument, we shall suppress the first two arguments in what follows.

THEOREM 5. Let $F$ be real-valued and continuous on $\wedge^{\nu} E$. Then a necessary and sufficient condition that $F \circ \Lambda$ be quasi-convex is that $F \mid \wedge^{\nu} T$ be convex for each $(\nu+1)$-dim subspace $T$ of $E$.

Proof. Suppose that $f=F \circ \Lambda$ is quasi-convex and that $T$ is a $(\nu+1)$-dim subspace of $E$. Let $\rho$ and $\sigma$ be linearly independent elements of $\Lambda^{\nu} T$ and let $t \in(0,1)$. Since $\operatorname{dim} T=\nu+1 \rho$ and $\sigma$ are necessarily simple and there exist $\nu$-planes $R$ and $S$ in $T$ associated with $\rho$ and $\sigma$. Furthermore, $R \cap S$ is a $(\nu-1)$-plane. Let $p_{1}, \cdots$, $p_{\nu-1}$ be a basis for $R \cap S$. There exist $r$ and $s$ in $T$ such that $\rho=$ $p_{1} \wedge \cdots \wedge p_{\nu-1} \wedge r$ and $\sigma=p_{1} \wedge \cdots \wedge p_{\nu-11} \wedge s$. Let $\lambda \in R_{\nu}, \quad \lambda=$ $(0, \cdots, 0,1)$ and let $\mu=-\lambda$. Let $\xi=(r-s) / 2$ and let $p_{\nu}=r-\xi=$ $s+\xi$. Then

$$
\begin{aligned}
t \rho+(1-t) \sigma & =p_{1} \wedge \cdots \wedge p_{\nu-1} \wedge\left(t\left(p_{\nu}+\xi\right)+(1-t)\left(p_{\nu}-\xi\right)\right. \\
& =\Lambda(p+(t \lambda+(1-t) \mu) \xi)
\end{aligned}
$$

so that

$$
\begin{aligned}
F(t \rho+(1-t) \sigma) & =f(p+(t \lambda+(1-t) \mu) \xi) \\
& \leqq t f(p+\lambda \xi)+(1-t) f(p+\mu \xi) \\
& =t F(\rho)+(1-t) F(\sigma) .
\end{aligned}
$$

To prove sufficiency let $p_{1}, \cdots, p_{\nu}$ and $\xi$ be in $E$ so that $p=$ $\left(p_{1}, \cdots, p_{\nu}\right)$ can be interpreted as an element of $P$ and let $T$ be a $(\nu+1)$-dim subspace of $E$ containing all these points. Let $\lambda$ and $\mu \in R_{\nu}$ and $t \in(0,1)$. Since $\xi \wedge \xi=0$,

$$
\Lambda(p+(t \lambda+(1-t) \mu) \xi)=t \Lambda(p+\lambda \xi)+(1-t) \Lambda(p+\mu \xi) .
$$

Hence

$$
\begin{aligned}
f(p+(t \lambda+(1-t) \mu) \xi) & \leqq t F(\Lambda(p+\lambda \xi))+(1-t) F(\Lambda(p+\mu \xi)) \\
& =t f(p+\lambda \xi)+(1-t) f(p+\mu \xi) .
\end{aligned}
$$




\section{REFERENCES}

1. H. Busemann and G. C. Shephard, Convexity on nonconvex sets, Proceedings of the colloquium on convexity, Copenhagen (1965).

2. C. B. Morrey, Jr. Quasi-convexity and the lower semicontinuity of multiple integrals, Pacific J. Math., 2 (1952), 25-53.

3. Multiple Integrals in the Calculus of Variations, New York, Springer (1966).

Received April 28, 1972. This research was partially supported by US-AFOSR 69-1662.

Purdue University 


\section{PACIFIC JOURNAL OF MATHEMATICS}

\section{EDITORS}

D. Gilbarg and J. Milgram

Stanford University

Stanford, California 94305

\author{
R. A. Beaumont \\ University of Washington \\ Seattle, Washington 98105
}

J. DuGUNDJI

Department of Mathematics

University of Southern California

Los Angeles, California 90007

Richard ARENS

University of California

Los Angeles, California 90024

\section{ASSOCIATE EDITORS}
E. F. BECKENBACH
B. H. NeumanN
F. WOLF
K. YosHIDA

\section{SUPPORTING INSTITUTIONS}

UNIVERSITY OF BRITISH COLUMBIA

CALIFORNIA INSTITUTE OF TECHNOLOGY

UNIVERSITY OF CALIFORNIA

MONTANA STATE UNIVERSITY

UNIVERSITY OF NEVADA

NEW MEXICO STATE UNIVERSITY

OREGON STATE UNIVERSITY

UNIVERSITY OF OREGON

OSAKA UNIVERSITY

\author{
UNIVERSITY OF SOUTHERN CALIFORNIA \\ STANFORD UNIVERSITY \\ UNIVERSITY OF TOKYO \\ UNIVERSITY OF UTAH \\ WASHINGTON STATE UNIVERSITY \\ UNIVERSITY OF WASHINGTON
* * * *
AMERICAN MATHEMATICAL SOCIETY \\ NAVAL WEAPONS CENTER
}

The Supporting Institutions listed above contribute to the cost of publication of this Journal, but they are not owners or publishers and have no responsibility for its content or policies.

Mathematical papers intended for publication in the Pacific Journal of Mathematics should be in typed form or offset-reproduced, (not dittoed), double spaced with large margins. Underline Greek letters in red, German in green, and script in blue. The first paragraph or two must be capable of being used separately as a synopsis of the entire paper. The editorial "we" must not be used in the synopsis, and items of the bibliography should not be cited there unless absolutely necessary, in which case they must be identified by author and Journal, rather than by item number. Manuscripts, in duplicate if possible, may be sent to any one of the four editors. Please classify according to the scheme of Math. Rev. Index to Vol. 39. All other communications to the editors should be addressed to the managing editor, Richard Arens, University of California, Los Angeles, California, 90024.

50 reprints are provided free for each article; additional copies may be obtained at cost in multiples of 50 .

The Pacific Journal of Mathematics is issued monthly as of January 1966. Regular subscription rate: $\$ 48.00$ a year (6 Vols., 12 issues). Special rate: $\$ 24.00$ a year to individual members of supporting institutions.

Subscriptions, orders for back numbers, and changes of address should be sent to Pacific Journal of Mathematics, 103 Highland Boulevard, Berkeley, California, 94708.

PUBLISHED BY PACIFIC JOURNAL OF MATHEMATICS, A NON-PROFIT CORPORATION

Printed at Kokusai Bunken Insatsusha (International Academic Printing Co., Ltd.), 270, 3-chome Totsuka-cho, Shinjuku-ku, Tokyo 160, Japan. 


\section{Pacific Journal of Mathematics}

\section{Vol. 46, No. 2 December, 1973}

Christopher Allday, Rational Whitehead products and a spectral sequence of

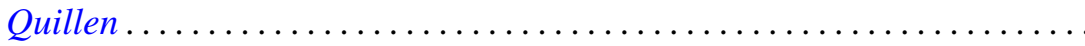

James Edward Arnold, Jr., Attaching Hurewicz fibrations with fiber

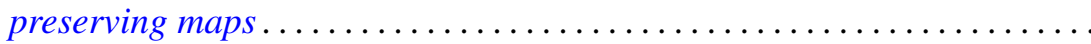

Catherine Bandle and Moshe Marcus, Radial averaging transformations with various metrics.................................

David Wilmot Barnette, A proof of the lower bound conjecture for convex

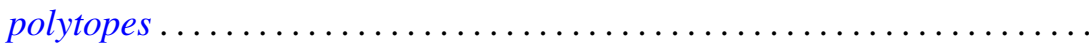

Louis Harvey Blake, Simple extensions of measures and the preservation of

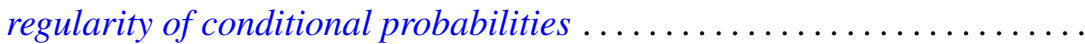

James W. Cannon, New proofs of Bing's approximation theorems for

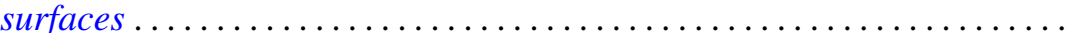

C. D. Feustel and Robert John Gregorac, On realizing HNN groups in

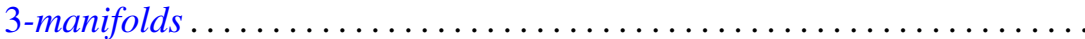

Theodore William Gamelin, Iversen's theorem and fiber algebras . . . . . . . . 389

Daniel H. Gottlieb, The total space of universal fibrations . . . . . . . . . . . .

Yoshimitsu Hasegawa, Integrability theorems for power series expansions of

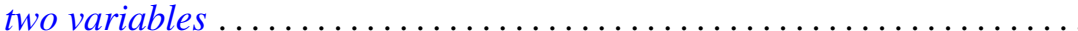

Dean Robert Hickerson, Length of period simple continued fraction expansion of $\sqrt{ } d$

Herbert Meyer Kamowitz, The spectra of endomorphisms of the disc algebra.

Dong S. Kim, Boundedly holomorphic convex domains

Daniel Ralph Lewis, Integral operators on $\mathscr{L}_{p}$-spaces ...

John Eldon Mack, Fields of topological spaces . . . . . . . . .

V. B. Moscatelli, On a problem of completion in bornology

Ellen Elizabeth Reed, Proximity convergence structures. .

Ronald C. Rosier, Dual spaces of certain vector sequence spaces .

Robert A. Rubin, Absolutely torsion-free rings

Leo Sario and Cecilia Wang, Radial quasiharmonic functions . .

James Henry Schmerl, Peano models with many generic classes .

H. J. Schmidt, The $\mathscr{F}$-depth of an $\mathscr{F}$-projector ............

Edward Silverman, Strong quasi-convexity. . . . . . . . . . . . . . . . . 549

Barry Simon, Uniform crossnorms ....................... 555

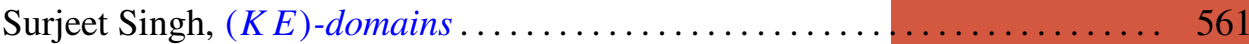

Ted Joe Suffridge, Starlike and convex maps in Banach spaces . . . . . . . . 575

Milton Don Ulmer, $C$-embedded $\Sigma$-spaces . . . . . . . . . . . . . . . . 591

Wolmer Vasconcelos, Conductor, projectivity and injectivity . . . . . . . . . 603 